ESAIM: PROCEEDINGS AND SURVEYS, February 2019, Vol. 65, p. 476-497

B. Bouchard, J.-F. Chassagneux, F. Delarue, E. Gobet and J. Lelong, Editors

\title{
SOME NON MONOTONE SCHEMES FOR HAMILTON-JACOBI-BELLMAN EQUATIONS
}

\author{
XAVIER WARIN ${ }^{1}$
}

\begin{abstract}
We extend the theory of Barles Jakobsen [3] for a class of almost monotone schemes to solve stationary Hamilton Jacobi Bellman equations. We show that the monotonicity of the schemes can be relaxed still leading to the convergence to the viscosity solution of the equation even if the discrete problem can only be solved with some error. We give some examples of such numerical schemes and show that the bounds obtained by the framework developed are not tight. At last we test the schemes.

Résumé. La théorie de Barles Jakobsen [3] pour la résolution des équations d'Hamilton Jacobi Bellman stationnaires est étendue pour une classe de schémas presque linéaires. Nous montrons que la monotonicité du schéma peut être relaxée tout en assurant la convergence vers la solution de viscosité du problème de contrôle bien que le problème discret ne puisse être résolu exactement. Nous donnons des exemples de schémas numériques entrant dans le cadre développé et montrons que les bornes obtenues ne sont pas optimales. Enfin nous testons ces schémas.
\end{abstract}

\section{INTRODUCTION}

We are interested in the following HJB equation arising in infinite horizon, discounted, stochastic control problems

$$
F\left(x, u, \mathbf{D} u, \mathcal{D}^{2} u\right)=0 \text { in } \mathbb{R}^{N},
$$

with

$$
\begin{aligned}
F(x, t, p, X) & =\sup _{\alpha \in \mathcal{A}} \mathcal{L}^{\alpha}(x, t, p, X), \\
\mathcal{L}^{\alpha}(x, t, p, X) & =-\operatorname{tr}\left[a^{\alpha}(x) X\right]-b^{\alpha}(x) p+c^{\alpha}(x) t-f^{\alpha}(x) .
\end{aligned}
$$

where $a, b, c, f$ are at least continuous functions on $\mathbb{R}^{N} \times \mathcal{A}$ with values in $S(N)$ the space of symmetric $N \times N$ matrices, $\mathbb{R}^{N}, \mathbb{R}$ and $\mathbb{R}$ respectively. The space of controls $\mathcal{A}$ is supposed to be a compact metric space.

Supposing $h$ is an approximating parameter, we consider an approximation $S$ of $F$ such that the approximate function $u_{h}$ satisfies:

$$
S\left(h, x, u_{h}(x),\left[u_{h}\right]_{x}\right)=0, x \in \mathbb{R}^{N}
$$

where $S\left(h, x, r,[t]_{x}\right)$ is defined for $x \in \mathbb{R}^{N}, r \in \mathbb{R}, t$ a function defined on $\mathbb{R}^{N}$, and $[t]_{x}$ is a function defined at $x$ from $t$. This notation was introduced by [5] to prove that a scheme $S$ which is non decreasing in $r$ and

\footnotetext{
${ }^{1}$ EDF R\&D \& FiME, Laboratoire de Finance des Marchés de l'Energie; e-mail: $\quad$ xavier.warin@edf.fr
}

(C) EDP Sciences, SMAI 2019 
non increasing in $[t]_{x}$ is monotone. When the scheme $S$ is a monotone, uniformly continuous and a consistent approximation of $F$ and when a discrete bounded solution $u_{h}$ can be found for (3), then $u_{h}$ converges to the viscosity solution of the problem (1) [3,4]. It is also possible to modify the consistency version proposed by [5] and still get a scheme converging to the viscosity solution for example for some finite element schemes defined in $[17]$.

When the scheme is non monotone, some theory exists to get convergent schemes in Sobolev space [31] but breaking monotonicity can lead to non converging schemes as shown in [25], and [28] gave some examples of non monotone schemes converging toward a false solution.

Methods to solve HJB equations includes Finite Difference methods and Semi Lagrangian methods. Classical Finite Difference method often can be interpreted as a Markov Chain [21] leading to monotone schemes. When $a^{\alpha}$ is not diagonally dominant, the requirement about monotonicity of the scheme leads to Finite Difference scheme such as in [10] using ideas independently developed in [19]. As an alternative to Finite Difference schemes, Semi Lagrangian schemes of low order based on the original work in [24] have been developed in [4,11]. Monotonicity of the schemes is desirable because a convenient framework is available. Nevertheless, it is to notice that some high order non monotone schemes have been developed and proved convergent for some first order Hamilton-Jacobi equations for examples in [2,23] or in [22] using spectral methods on a periodic domain. In the case of first order Hamilton-Jacobi-Bellman equation, some non monotone explicit schemes were developed because of the inefficiency of monotone scheme for discontinuous initial data and proved to be convergent in [8].

In this paper we will relax too the constraint on the monotonicity of the scheme such that it can converge to the right solution. We will suppose that this scheme is a perturbation of a monotone scheme $\hat{S}$ and we have in mind the schemes based on interpolation method (Semi Lagrangian scheme or Finite Difference scheme with carefully chosen directions). This idea is not new : [5] already have emphasized the fact that monotonicity could be relaxed. As for Finite Difference schemes, for first order Hamilton-Jacobi, following the ideas in [1,16], some potentially high order scheme dubbed filtered schemes have been developed in $[7,26]$ by blending two schemes: one of high order potentially instable and one monotone of low order that will be used near singularity of the solution. Some filtered schemes have been also used for second order HJB equations for example in [9]. As for Semi Lagrangian, results for high order schemes interpolators are given by the Italian School for first order Hamilton-Jacobi in [15]. The interest in nearly monotone scheme is driven by the fact that monotone scheme such as Semi-Lagrangian schemes with linear interpolators are converging numerically very slowly. Then it seems natural to try to use schemes that are potentially fast convergent (with potentially a high order of consistency) while being sure that they converge towards the true solution even if we cannot prove a higher rate of convergence than in the monotone case. Our goal is to develop a framework that could be used to develop new schemes and easily prove that they are convergent.

Specifically we treat the second order Hamilton Jacobi Bellman stationary problem with nearly monotone schemes. Because in some case the resolution of (3) can be impossible we will try to relax the equality requirement and only assume we can find a function $u_{h}$ such that on a given grid $X$ that may depend on $h$

$$
\left|S\left(h, x, u_{h}(x),\left[u_{h}\right]_{x}\right)\right| \leq \epsilon(h), x \in X
$$

where $\epsilon(h)$ is a continuous function of $h$ with $\epsilon(0)=0$.

If existing, the solution of such a scheme is not unique, so we consider a constructed sequence of solution $u_{h}$ of (4) and get bounds proving the convergence of $u_{h}$ towards the right solution.

This work is motivated by the previous work by the author [32] : some almost monotone Semi Lagrangian schemes with high order interpolation and truncation were developed in the time dependent case. It was proven that such schemes could be used to estimate the solution of time dependent Hamilton-Jacobi-Bellman equations. A numerical study was achieved comparing different types of Lagrange interpolators, Bernstein approximations, and Cubic spline interpolators. The conclusion was that Gauss Lobatto Legendre (GLL) interpolators were the most interesting given the accuracy obtained compared to the CPU time used.

In the stationary case classical techniques involving fixed point iteration scheme cannot be used to proved 
existence of a solution of the discretized problem: it leads to the idea of relaxation given by equation (4) and to the development of a general framework.

In a second part of the article, we detail the semi-lagrangian schemes proposed by [32] and show that they can be cast into this framework so that some convergence properties can be derived. In the present article, we only focus on GLL interpolators to study the stationary Hamilton-Jacobi-Bellman equations in our framework. Using a direct estimation, we besides prove that the result obtained by the framework is not optimal. In fact, with the direct estimation, we get back the convergence result in $O\left(h^{\frac{1}{4}}\right)$ previously obtained in $[4,11]$.

We then develop a Finite Difference approach with interpolation. In order to use the developed framework, we have to suppose that the diffusion coefficient is independent of the space. Once again the method can be cast in the framework developed and the convergence rate obtained is not optimal. The best rate we found is in $O\left(h^{\frac{1}{2}}\right)$ which is the rate found in [4] with the same assumptions. As for the general case where the diffusion depends on the space, the same rate of convergence is reached using the Bonnans and al. [10] or Krylov [19] discretization as shown in [19].

In the sequel the constant $C$ may vary between lines.

\section{MAIN RESUlT}

We define the norm denoted $\|$ as follows: for any integer $m \geq 1$ and $z=\left(z_{i}\right)_{i} \in \mathbb{R}^{m}$, we set $|z|^{2}=\sum_{i=1}^{m} z_{i}^{2}$. For a matrix $M \in \mathbb{R}^{n_{1} \times n_{2}},|M|^{2}=\operatorname{tr}\left[M^{t} M\right]$ with $M^{t}$ the transpose of $M$.

If $f: \mathbb{R}^{N} \longrightarrow \mathbb{R}^{M}$ we define the semi-norms:

$$
|f|_{0}=\sup _{x \in \mathbb{R}^{N}}|f(x)|, \quad[f]_{1}=\sup _{\substack{x, y \in \mathbb{R}^{N} \\ x \neq y}} \frac{|f(x)-f(y)|}{|x-y|},
$$

and

$$
|f|_{1}=|f|_{0}+[f]_{1}
$$

$C^{0,1}\left(\mathbb{R}^{N}\right)$ stands for the set of functions $f: \mathbb{R}^{n} \longrightarrow \mathbb{R}$ with finite norm $|f|_{1}, C_{b}\left(\mathbb{R}^{N}\right)$ the set with finite norm $|f|_{0}$. In the sequel we make the following classical assumptions

Assumption (A1). For any $\alpha \in \mathcal{A}, a^{\alpha}=\frac{1}{2} \sigma^{\alpha} \sigma^{\alpha t}$ for some $N \times P$ matrix $\sigma^{\alpha}$. Furthermore, there exists $\lambda, K$ independent of $\alpha$ such that:

$$
c^{\alpha} \geq \lambda>0, \text { and }\left|\sigma^{\alpha}\right|_{1}+\left|b^{\alpha}\right|_{1}+\left|f^{\alpha}\right|_{1} \leq K .
$$

Assumption (A2). The constant $\lambda$ in (A1) satisfies $\lambda>\sup _{\alpha} \frac{1}{2}\left[\sigma^{\alpha}\right]_{1}^{2}+\left[b^{\alpha}\right]_{1}$.

We just recall the well-posedness and regularity result given in [4] with demonstrations in the references therein.

Proposition 1.1. Assume (A1): There exists a unique viscosity u solution of (1) in $C_{b}\left(\mathbb{R}^{N}\right)$. If $w_{1}$ and $w_{2}$ are in $C_{b}\left(\mathbb{R}^{N}\right)$ and are sub- and supersolution of (1) respectively, then $w_{1} \leq w_{2}$ in $\mathbb{R}^{N}$.

Assume (A1), (A2): There exists a unique bounded viscosity $u$ solution of (1) in $C^{0,1}\left(\mathbb{R}^{N}\right)$.

Remark 1.2. Assumptions (A1) can be given for more general Hölder spaces, and regularity of the solution is then given in [3].

Here we add some new definitions that will be helpful in the sequel. First we introduce the notion of $\epsilon$ monotone scheme stating that the scheme $S$ is "nearly" monotone 
Definition 1.3. An $\epsilon(p, K)$ monotone scheme $S$ is a scheme such that there exists $\bar{\lambda}$ satisfying:

- for every $h>0, x \in \mathbb{R}^{N}, r \in \mathbb{R}$, for every function $w \in C^{0,1}\left(\mathbb{R}^{N}\right), v \in C_{b}\left(\mathbb{R}^{N}\right)$ such that $v \geq w$ :

$$
S\left(h, x, r,[v]_{x}\right) \leq S\left(h, x, r,[w]_{x}\right)+K|w|_{1} h^{p}
$$

- for every $h>0, x \in \mathbb{R}^{N}, r \in \mathbb{R}$, for every function $w \in C_{b}\left(\mathbb{R}^{N}\right), v \in C^{0,1}\left(\mathbb{R}^{N}\right)$ such that $v \geq w$ :

$$
S\left(h, x, r,[v]_{x}\right) \leq S\left(h, x, r,[w]_{x}\right)+K|v|_{1} h^{p}
$$

- for every $h>0, x \in \mathbb{R}^{N}, r \in \mathbb{R}, m \geq 0$, for every function $u \in C_{b}\left(\mathbb{R}^{N}\right)$ :

$$
S\left(h, x, r+m,[u+m]_{x}\right) \geq S\left(h, x, r,[u]_{x}\right)+\bar{\lambda} m .
$$

Because it is sometimes difficult or impossible to prove that a discrete scheme has a solution, we relax the notion of solution as we did in (4):

Definition 1.4. Relaxing the notion of solution, subsolution and supersolution we introduce:

- An $\epsilon(c)$ solution $u$ of scheme $S$ is a continuous function which satisfies:

$$
\left|S\left(h, x, u(x),[u]_{x}\right)\right|<c .
$$

- An $\epsilon(c)$ subsolution (supersolution) $u$ of scheme $S$ is a continuous function which satisfies:

$$
S\left(h, x, u(x),[u]_{x}\right)<c \quad\left(S\left(h, x, u(x),[u]_{x}\right)>-c\right) .
$$

On the scheme we make the following assumptions:

Assumption (S1). The scheme $S$ is $\epsilon(p, K)$ monotone.

Assumption (S2). (Regularity of $S$ scheme) For every $h>0$ and $\phi \in C_{b}\left(\mathbb{R}^{N}\right), x \longrightarrow S\left(h, x, \phi(x),[\phi]_{x}\right)$ is bounded and continuous in $\mathbb{R}^{N}$ and the function $r \longrightarrow S\left(h, x, r,[\phi]_{x}\right)$ is uniformly continuous for bounded $r$, uniformly in $x \in \mathbb{R}^{N}$.

Assumption (S3). (Consistency) There exists a set of strictly positive integers $\left(k_{i}\right)_{i=1, m}$, and a constant $K$ such that for every $h \geq 0, x \in \mathbb{R}^{N}$ and a smooth function $\phi$

$$
\left|F\left(x, \phi(x), \mathcal{D} \phi(x), \mathcal{D}^{2} \phi\right)-S\left(h, x, \phi(x),[\phi]_{x}\right)\right| \leq K \sum_{i=1}^{m} h^{k_{i}}\left|\mathcal{D}^{i} \phi\right|_{0} .
$$

We add an assumption on the existence of a solution of the discretized scheme with sufficient regularity which has to be checked for each scheme:

Assumption (S4). We suppose there exists $C$ and $r$ independent of $h$ such that for each $h$ we can find an $\epsilon\left(C h^{r}\right)$ solution $u_{h}$ of scheme $S$.

Remark 1.5. Assumptions (S2) and (S3) are classical. Assumption (S1) is a relaxation of the constraint on the monotonicity of the scheme.

Remark 1.6. Related to assumption (S4), [6] studied the case where some non monotone schemes were solved within some margin error.

An $\epsilon$ monotone scheme doesn't ensure the existence of a discrete comparison result but we give here a relaxation of this result: 
Lemma 1.7. Assume (S1). Let $v \in C_{b}\left(\mathbb{R}^{N}\right)$ and $u \in C^{0,1}\left(\mathbb{R}^{N}\right)$. If $u$ is a subsolution of (4) and $v$ is an $\epsilon(C)$ supersolution of (4) then

$$
u \leq v+\frac{1}{\bar{\lambda}}\left(K h^{p}|u|_{1}+C\right) .
$$

Proof. Mimicking Lemma 2.3 in [4], we assume $m:=\sup _{\mathbb{R}^{N}}(u-v)>\frac{1}{\lambda}\left(K h^{p}|u|_{1}+C\right)$. Let $\left\{x_{n}\right\}_{n}$ be a sequence such that $u\left(x_{n}\right)-v\left(x_{n}\right):=\delta_{n} \longrightarrow m$. For $n$ large enough, $\delta_{n}>\frac{1}{\lambda}\left(K h^{p}|u|_{1}+C\right)$. Using the subsolution definition, assumption (S1) :

$$
\begin{aligned}
& 0 \geq S\left(h, x_{n}, u\left(x_{n}\right),[u]_{x_{n}}\right)-S\left(h, x_{n}, v\left(x_{n}\right),[v]_{x_{n}}\right)-C, \\
& 0 \geq S\left(h, x_{n}, v\left(x_{n}\right)+\delta_{n},[v+m]_{x_{n}}\right)-S\left(h, x_{n}, v\left(x_{n}\right),[v]_{x_{n}}\right)-K h^{p}|u|_{1,0}-C, \\
& 0 \geq \bar{\lambda} \delta_{n}+w\left(m-\delta_{n}\right)-K h^{p}|u|_{1}-C,
\end{aligned}
$$

where $w(t) \longrightarrow 0$ when $t^{+} \longrightarrow 0$ by assumption (S2). Letting $n \longrightarrow \infty$, we get

$$
m \leq \frac{1}{\bar{\lambda}}\left(K h^{p}|u|_{1}+C\right),
$$

which gives the contradiction.

We now give the lower and upper bound for the error given by the scheme. The lower bound will be given by the classical Krylov method of shaking coefficients [20], while the upper bound will be given by the use of a switching system as in $[3,14]$ that gives a supersolution of the problem. To use the theory developed in [3], we need to add one last assumption:

Assumption (A3). For every $\delta>0$, there are $Q \in \mathbb{N}$ and $\left\{\alpha_{i}\right\}_{i=1}^{Q} \subset \mathcal{A}$, such that for any $\alpha \in \mathcal{A}$

$$
\inf _{1 \leq i \leq Q}\left(\left|\sigma^{\alpha}-\sigma^{\alpha_{i}}\right|_{0}+\left|b^{\alpha}-b^{\alpha_{i}}\right|_{0}+\left|c^{\alpha}-c^{\alpha_{i}}\right|_{0}+\left|f^{\alpha}-f^{\alpha_{i}}\right|_{0}\right)<\delta .
$$

We introduce the following switching system:

$$
F_{i}^{\epsilon}\left(x, v^{\epsilon}, \mathcal{D} v_{i}^{\epsilon}, \mathcal{D}^{2} v_{i}^{\epsilon}\right)=0 \text { in } \mathbb{R}^{N}, i \in \mathcal{I}:=\{1, . ., Q\},
$$

where $v^{\epsilon}=\left(v_{1}^{\epsilon}, \ldots, v_{Q}^{\epsilon}\right)$,

$$
F_{i}^{\epsilon}(x, r, p, X)=\max \left\{\min _{|e| \leq \epsilon} \mathcal{L}^{\alpha_{i}}\left(x+e, r_{i}, p, X\right) ; r_{i}-\mathcal{M}_{i} r\right\}
$$

and $\mathcal{L}^{\alpha}$ given by (2),

$$
\mathcal{M}_{i} r=\min _{j \neq i}\left\{r_{j}+k\right\} .
$$

We give two lemmas proved in [3]:

Lemma 1.8. Assume (A1) and (A2).

- There exists a unique solution $v^{\epsilon}$ of (9) satisfying $\left|v^{\epsilon}\right|_{1} \leq C$ where $C$ depends only on $\lambda, K$ from (A1).

- Assume in addition (A3), then for any $\delta>0$, there are $Q \in \mathbb{N}$ and $\left\{\alpha_{i}\right\}_{i=1}^{Q} \subset \mathcal{A}$ such that the solution $v_{\epsilon}$ of (9) satisfies

$$
\max _{i}\left|u-v_{i}^{\epsilon}\right|_{0} \leq C\left(\epsilon+k^{\frac{1}{3}}+\delta\right),
$$

where $C$ depends on $\lambda, K$ from (A1). 
A function $u$ can be regularized by

$$
\rho_{\epsilon} * u(x):=\int_{\mathbb{R}^{N}} u(x-e) \rho_{\epsilon}(e) d e,
$$

where $\rho_{\epsilon}$ is the mollifier sequence such that

$$
\rho_{\epsilon}(x)=\frac{1}{\epsilon^{N}} \rho\left(\frac{x}{\epsilon}\right) \text { where } \rho \in C^{\infty}\left(\mathbb{R}^{N}\right), \int_{\mathbb{R}^{N}} \rho=1 \text {, and } \operatorname{supp}(\rho)=\bar{B}(0,1) .
$$

Lemma 1.9. Assume (A1) and (A2) and define $v_{\epsilon, i}:=\rho_{\epsilon} * v_{i}^{\epsilon}$ for $i \in \mathcal{I}$.

- There is a constant $C$ depending only on $\lambda, K$ from (A1), such that

$$
\left|v_{\epsilon, j}-v_{i}^{\epsilon}\right|_{0} \leq C(k+\epsilon) \text { for } i, j \in \mathcal{I} \text {. }
$$

- Assume in addition that $\epsilon \leq\left(4 \sup _{i}\left[v_{i}^{\epsilon}\right]_{1}\right)^{-1} k$. For every $x \in \mathbb{R}^{N}$, if $j:=\operatorname{argmin}_{i \in \mathcal{I}} v_{\epsilon, i}(x)$, then

$$
\mathcal{L}^{\alpha_{j}}\left(x, v_{\epsilon, j}(x), \mathcal{D} v_{\epsilon, j}(x), \mathcal{D}^{2} v_{\epsilon, j}(x)\right) \geq 0 .
$$

We can now give the main result of the paper using the two previous lemmas for an upper bound of the $\epsilon$ solution.

Theorem 1.10. Assume (A1), (A2), (S1), (S2), (S3), and (S4). We have the following bounds for $u_{h}$ a sequence of $\epsilon\left(\tilde{C} h^{r}\right)$ solutions:

$$
u-u_{h} \leq \hat{C}\left(h^{\min (p, r)}+h^{i=1, m} \min ^{\frac{k_{i}}{i}}\right),
$$

where $\hat{C}$ depends on $\tilde{C}$.

Besides assume (A3) then there exists $\hat{C}$ depending on $\tilde{C}$ such that :

$$
u_{h}-u \leq \hat{C}\left(h^{\min (p, r)}+h^{\min ^{i=1, m} \frac{k_{i}}{3 i-2}}\right)
$$

Proof. For the lower bound, we follow the Krylov demonstration [20] as done in [4]. First we introduce the solution $u^{\epsilon}$

$$
\max _{|e| \leq \epsilon}\left[F\left(x+e, u^{\epsilon}, \mathcal{D} u^{\epsilon}, \mathcal{D}^{2} u^{\epsilon}\right)\right]=0 \text { in } \mathbb{R}^{N}
$$

The existence a solution $u^{\epsilon}$ in $C^{0,1}\left(\mathbb{R}^{N}\right)$ satisfying $\left|u^{\epsilon}\right| \leq C$ and $\left|u^{\epsilon}-u\right|_{0} \leq C \epsilon$ is given by Lemma 2.6 in [4]. Noting that for each $e \leq \epsilon, u^{\epsilon}(.-e)$ satisfies for each function $\phi=\psi(.-e) \in C^{2}\left(\mathbb{R}^{N}\right)$ and each $y$ where $u^{\epsilon}(y-e)-\phi(y)$ is maximal

$$
\begin{aligned}
F\left(y, u^{\epsilon}(y-e), \mathcal{D} \phi(y), \mathcal{D}^{2} \phi(y)\right) & =F\left(y, u^{\epsilon}(y-e), \mathcal{D} \psi(y-e), \mathcal{D}^{2} \psi(y-e)\right) \\
& \leq \sup _{|e| \leq \epsilon} F\left(y, u^{\epsilon}(y-e), \mathcal{D} \psi(y-e), \mathcal{D}^{2} \psi(y-e)\right) \\
& \leq 0
\end{aligned}
$$

so $u^{\epsilon}(.-e)$ is a subsolution of $(1)$.

Then $u^{\epsilon}(.-e)$ is regularized by

$$
u_{\epsilon}(x):=\int_{\mathbb{R}^{N}} u^{\epsilon}(x-e) \rho_{\epsilon}(e) d e .
$$


The regularized function $u_{\epsilon}$ is a subsolution of problem (1) as given by Lemma 2.7 in [4]. First use the relation for $m>0$,

$$
F(x, t+m, p, X) \geq F(x, t, p, X)+\lambda m,
$$

and the consistency property (S3) to get

$$
\begin{aligned}
F\left(y, u_{\epsilon}(y), \mathcal{D} u_{\epsilon}(y), \mathcal{D}^{2} u_{\epsilon}(y)\right) \geq & F\left(y, u_{\epsilon}(y)-\frac{K}{\lambda} \sum_{i=1}^{m} h^{k_{i}}\left|\mathcal{D}^{i} u_{\epsilon}\right|_{0}, \mathcal{D} u_{\epsilon}(y), \mathcal{D}^{2} u_{\epsilon}(y)\right)+ \\
& K \sum_{i=1}^{m} h^{k_{i}}\left|\mathcal{D}^{i} u_{\epsilon}\right|_{0}, \\
\geq & S\left(h, y, u_{\epsilon}(y)-\frac{K}{\lambda} \sum_{i=1}^{m} h^{k_{i}}\left|\mathcal{D}^{i} u_{\epsilon}\right|_{0},\left[u_{\epsilon}-\frac{K}{\lambda} \sum_{i=1}^{m} h^{k_{i}}\left|\mathcal{D}^{i} u_{\epsilon}\right|_{0}\right]_{y}\right) .
\end{aligned}
$$

Then $u_{\epsilon}-\frac{K}{\lambda} \sum_{i=1}^{m}\left|\mathcal{D}^{i} u_{\epsilon}\right|_{0}$ is a subsolution of equation (4). Using lemma 1.7, and assumption (S4), we get that there exists $C$ such that

$$
\begin{aligned}
u_{\epsilon}-u_{h} & \leq C\left(\left|u_{\epsilon}\right|_{1} h^{p}+\sum_{i=1}^{m}\left|\mathcal{D}^{i} u_{\epsilon}\right|_{0} h^{k_{i}}+h^{r}\right), \\
& \leq C\left(h^{\min (p, r)}+\sum_{i=1}^{m} \epsilon^{1-i} h^{k_{i}}\right) .
\end{aligned}
$$

In the last line we have used that because $u^{\epsilon}$ is bounded uniformly in $C^{0,1}\left(\mathbb{R}^{N}\right), u_{\epsilon}$ is regular and $\left|\mathcal{D}^{n} u_{\epsilon}\right|_{0} \leq$ $C \epsilon^{1-n}$ for $n \geq 1$.

At last using the mollifier properties, the uniform boundedness of $u^{\epsilon}$ in $C^{0,1}$ gives us that $\left|u_{\epsilon}-u^{\epsilon}\right| \leq C \epsilon$. Besides $\left|u^{\epsilon}-u\right|_{0} \leq C \epsilon$ (Lemma 2.6 in [4]) so there exists $\hat{C}$ depending on $\tilde{C}$ such that

$$
\begin{aligned}
u-u_{h} & \leq\left|u-u^{\epsilon}\right|_{0}+\left|u^{\epsilon}-u_{\epsilon}\right|_{0}+u_{\epsilon}-u_{h}, \\
& \leq C\left(h^{\min (p, r)}+\sum_{i=1}^{m} \epsilon^{1-i} h^{k_{i}}+\epsilon\right) .
\end{aligned}
$$

Choosing $\epsilon=h^{\min _{i, m} \frac{k_{i}}{i}}$ we get the lower bound in the theorem.

For the upper bound we follow the Barles Jakobsen demonstration that we shorten except for points different from the initial proof. We fix a $\delta>0$, and pick up the corresponding $\left\{\alpha_{i}\right\}_{i \in \mathcal{I}}$ according to (A3). The corresponding solution $v^{\epsilon}$ of (9) exists according to lemma 1.8 and is regularized as in lemma 1.9. We note

$$
m:=\sup _{y \in \mathbb{R}^{N}}\left\{u_{h}(y)-w(y)\right\},
$$

where $w:=\min _{i \in \mathcal{I}} v_{\epsilon, i}$. We approximate $m$ by

$$
m_{\kappa}:=\sup _{y \in \mathbb{R}^{N}}\left\{u_{h}(y)-w(y)-\kappa \phi(y)\right\},
$$

where $\phi(y)=\left(1+|y|^{2}\right)^{\frac{1}{2}}$. Because of the boundedness and continuity of $u_{h}$ and $w$, the maximum is attained at a point $\mathrm{x}$. Because of the definition of $x$,

$$
m_{\kappa}:=\sup _{y \in \mathbb{R}^{N}}\left\{u_{h}(y)-v_{\epsilon, i}(y)-\kappa \phi(y)\right\},
$$


where $i=\operatorname{argmin}_{i \in \mathcal{I}} v_{\epsilon, i}(x)$.

Taking $\epsilon=\left(4 \sup _{i}\left[v_{i}^{\epsilon}\right]_{1}\right)^{-1} k$, from lemma 1.9, the definition of $\phi$ and (A1) we get

$$
\sup _{\alpha \in \mathcal{A}} \mathcal{L}^{\alpha}\left(x,\left(v_{\epsilon, i}+\kappa \phi\right)(x), \mathcal{D}\left(v_{\epsilon, i}+\kappa \phi\right)(x), \mathcal{D}^{2}\left(v_{\epsilon, i}+\kappa \phi\right)(x)\right) \geq-C \kappa .
$$

Then using (S3):

$$
-C \kappa \leq S\left(h,\left(v_{\epsilon, i}+\kappa \phi\right)(x),\left[v_{\epsilon, i}+\kappa \phi\right]_{x}\right)+K \sum_{j=1}^{m} h^{k_{j}}\left|\mathcal{D}^{j}\left(v_{\epsilon, i}+\kappa \phi\right)\right|_{0} .
$$

Using the properties of the mollified $v_{\epsilon, i}$, and the definition of $\phi$ :

$$
-K \sum_{j=1}^{m} h^{k_{j}} \epsilon^{1-j}+\mathcal{O}(\kappa) \leq S\left(h,\left(v_{\epsilon, i}+\kappa \phi\right)(x),\left[v_{\epsilon, i}+\kappa \phi\right]_{x}\right) .
$$

Then we use the $\epsilon$ monotony property (8), the definition of $m_{\kappa}$, the fact that $v_{\epsilon, i}$ is bounded uniformly by the properties of mollifiers and lemma 1.8 to get

$$
\begin{aligned}
S\left(h,\left(v_{\epsilon, i}+\kappa \phi\right)(x),\left[v_{\epsilon, i}+\kappa \phi\right]_{x}\right) & \leq S\left(h, u_{h}(x)-m_{\kappa},\left[u_{h}-m_{\kappa}\right]_{x}\right)+K h^{p}\left|v_{\epsilon, i}+\kappa \phi\right|_{1}, \\
& \leq-\lambda m_{\kappa}+S\left(h, u_{h}(x),\left[u_{h}\right]_{x}\right)+C h^{p}(1+\kappa), \\
& \leq \tilde{C}(1+\kappa) h^{\min (r, p)}-\lambda m_{\kappa} .
\end{aligned}
$$

Using (14) and (15) we get

$$
\lambda m_{\kappa} \leq \tilde{C}(1+\kappa) h^{\min (r, p)}+K \sum_{j=1}^{m} h^{k_{j}} \epsilon^{1-j}+\mathcal{O}(\kappa)
$$

An estimate of $m$ is obtained by letting $\kappa$ goes to 0 . Then for any $y \in \mathbb{R}^{N}$,

$$
\begin{aligned}
u_{h}(y)-u(y) & \leq u_{h}(y)-v_{\epsilon, i}(y)+v_{\epsilon, i}(y)-u(y) \\
& \leq m+v_{\epsilon, i}(y)-u(y) .
\end{aligned}
$$

Using the lemma 1.8 and 1.9 we get

$$
u_{h}(y)-u(y) \leq \hat{C}\left(h^{\min (r, p)}+\sum_{i=1}^{m} h^{k_{i}} \epsilon^{1-i}+\epsilon+k+k^{\frac{1}{3}}+\delta\right),
$$

with $\hat{C}$ depending on $\tilde{C}$ and uniform in $y$. The conclusion is obtained by taking $\epsilon=h^{i=1, m^{\frac{3}{3 i-2}}}$, remembering that $k=\epsilon \sup _{i}\left[v_{i}^{\epsilon}\right]_{1}$ and letting $\delta$ going to 0 .

\section{Some NumericAl SCHEMES}

In this section we take the notations and we will follow arguments similar to [3,4]. We give the notations used for the discretization and interpolation used by the scheme. A thorough study of interpolation method for time dependent HJB equation can be found in [32]. All the schemes defined in [32] can be used : it includes truncated Lagrangian interpolators, classical cubic spline truncated interpolators, the monotone cubic spline first defined in [12]. In the sequel we focus on the truncated GLL interpolators which are the most effective according to [32]. 
A spatial discretization $\Delta x$ of the problem being given, in the sequel a mesh $\hat{X}_{\bar{i}}$ corresponds to the hyper-cube $\left[i_{1} \Delta x,\left(i_{1}+1\right) \Delta x\right] \times \ldots \times\left[i_{N} \Delta x,\left(i_{N}+1\right) \Delta x\right]$ with $\bar{i}=\left(i_{1}, \ldots, i_{N}\right) \in \mathbf{Z}^{N}$. For GLL quadrature grid $\left(\xi_{i}\right)_{i=1, \ldots, M+1} \in$ $[-1,1]$, with $\xi_{1}=-1, \xi_{M+1}=1$, and for a mesh $\bar{i}$, the point $y_{\bar{i}, \tilde{j}}$ with $\tilde{j}=\left(j_{1}, \ldots, j_{N}\right) \in[1, M+1]^{N}$ will have the coordinate $\left(\Delta x\left(i_{1}+0.5\left(1+\xi_{j_{1}}\right)\right), \ldots, \Delta x\left(i_{N}+0.5\left(1+\xi_{j_{N}}\right)\right)\right.$. We denote $X_{\Delta x, M}:=\left(y_{\bar{i}, \tilde{j}}\right)_{\bar{i}, \tilde{j}}$ the set of all the grids points on the whole domain.

We notice that each mesh $\hat{X}_{\bar{i}}$ has a constant volume $\Delta x^{N}$, so we have the following relation for all $x \in \mathbb{R}^{N}$ :

$$
\min _{\bar{i}, \tilde{j}}\left|x-y_{\bar{i}, \tilde{j}}\right| \leq C \Delta x
$$

We introduce $I_{\Delta x, M}$ the Lagrange interpolator associated to the GLL quadrature. We recall that in one dimension, the GLL Lagrange interpolator $I_{M}$ on $[-1,1]$ is given by (see for example [29]):

$$
\begin{aligned}
I_{M}(f) & =\sum_{k=0}^{M} \tilde{f}_{k} L_{k}(x), \\
\tilde{f}_{k} & =\frac{1}{\gamma_{k}} \sum_{i=0}^{M} \rho_{i} f\left(\eta_{i}\right) L_{k}\left(\eta_{i}\right), \\
\gamma_{k} & =\sum_{i=0}^{M} L_{k}\left(\eta_{i}\right)^{2} \rho_{i},
\end{aligned}
$$

where the functions $L_{N}$ satisfy the recurrence

$$
\begin{aligned}
(N+1) L_{N+1}(x) & =(2 N+1) x L_{N}(x)-N L_{N-1}(x), \\
L_{0} & =1, \quad L_{1}=x
\end{aligned}
$$

$\eta_{1}=-1, \eta_{M+1}=1$, the $\eta_{i}(i=2, \ldots, M)$ are the zeros of $L_{M}^{\prime}$ and the eigenvalues of the matrix $P$

$$
\begin{aligned}
P & =\left(\begin{array}{lllll}
0 & \gamma_{1} & \ldots & 0 & 0 \\
\gamma_{1} & 0 & \ldots & 0 & 0 \\
\ldots & \ldots & \ldots & \ldots & \ldots \\
0 & 0 & \ldots & 0 & \gamma_{M-2} \\
0 & 0 & \ldots & \gamma_{M-2} & 0
\end{array}\right), \\
\gamma_{n} & =\frac{1}{2} \sqrt{\frac{n(n+2)}{\left(n+\frac{1}{2}\right)\left(n+\frac{3}{2}\right)}}, 1 \leq n \leq M-2,
\end{aligned}
$$

and the weights satisfies

$$
\rho_{i}=\frac{2 .}{(M+1) M L_{M}^{2}\left(\eta_{i}\right)}, 1 \leq i \leq M+1 .
$$

The interpolator $I_{\Delta x, M}$ on a mesh $\hat{X}_{\bar{i}}$ is obtained by first rescaling $I_{M}$ and by tensorization.

On a mesh $\hat{X}_{\bar{i}}$, we note $\underline{v}_{\bar{i}}=\min _{\tilde{j}} v\left(y_{\bar{i}, \tilde{j}}\right), \bar{v}_{\bar{i}}=\max _{\tilde{j}} v\left(y_{\bar{i}, \tilde{j}}\right)$. We introduce the following truncated operator:

$$
\hat{I}_{\Delta x, M}(v)=\underline{v}_{\bar{i}} \vee I_{\Delta x, M}(v) \wedge \bar{v}_{\bar{i}},
$$

where $\wedge$ denotes the minimum and $\vee$ the maximum.

We first give some properties associated to the truncated interpolation operator. 
Lemma 2.1. The interpolator $\hat{I}_{\Delta x, M}$ satisfies

$$
\begin{aligned}
\left(\hat{I}_{\Delta x, M} f\right)(x) & =\sum_{\tilde{j}}\left(w_{\bar{i}, \tilde{j}}(f)\right)(x) f\left(y_{\bar{i}, \tilde{j}}\right) \\
\sum_{\tilde{j}}\left(w_{\bar{i}, \tilde{j}}^{h}(f)\right)(x) & =1,
\end{aligned}
$$

and the positive weights $w_{i, \tilde{j}}^{h}(f)$ are functions of $f$.

Proof. Because of the truncation for each point $x$ of a mesh $\hat{X}_{\bar{i}}$, we have

$$
\left(\hat{I}_{\Delta x, M}(f)(x)=\underline{w}_{\bar{i}}^{h}(f)(x) \underline{f}_{\bar{i}}+\bar{w}_{\bar{i}}^{h}(f)(x) \bar{f}_{\bar{i}} .\right.
$$

where the weights are defined as follows:

If $\underline{f}_{\bar{i}} \leq \hat{I}_{\Delta x, M}(f)(x) \leq \bar{f}_{\bar{i}}$ then

$$
\begin{aligned}
\underline{w}_{\bar{i}}^{h}(f)(x) & =\frac{\hat{I}_{\Delta x, M}(f)(x)-\bar{f}_{\bar{i}}}{\underline{f}_{\bar{i}}-\bar{f}_{\bar{i}}}, \\
\bar{w}_{\bar{i}}^{h}(f)(x) & =1-\underline{w}_{\bar{i}}^{h}(f)(x),
\end{aligned}
$$

If $\underline{f}_{\bar{i}}>\hat{I}_{\Delta x, M}(f)(x)$,

$$
\begin{aligned}
\bar{w}_{\bar{i}}^{h}(f)(x) & =0, \\
\underline{w}_{i}^{h}(f)(x) & =1 .
\end{aligned}
$$

Otherwise

$$
\begin{aligned}
& \underline{w}_{i}^{h}(f)(x)=0, \\
& \bar{w}_{\bar{i}}^{h}(f)(x)=1 .
\end{aligned}
$$

The weights associated to non extremal points are taken equal to 0 .

Then we add a result for the interpolation error :

Lemma 2.2. - For each K-Lipschitz bounded function $f$ :

$$
\left|\hat{I}_{\Delta x, M}(f)-f\right|_{0} \leq K(\Delta x) .
$$

- Suppose $M \geq 2$, for each $f \mathbb{R}$ value function defined on $\mathbb{R}^{N}$ and twice differentiable, there exists $C$ such that:

$$
\left|\hat{I}_{\Delta x, M}(f)-f\right|_{0} \leq C \Delta x^{2}\left|D^{2} f\right|_{0}
$$

- For $m \in R^{N}$,

$$
\hat{I}_{\Delta x, M}(f+m)(x)=\hat{I}_{\Delta x, M}(f)+m .
$$


Proof. First because of the truncation, continuity of $\hat{I}_{\Delta x, M}(f)$, for each $x \in \mathbb{R}^{N}, x \in \hat{X}_{\bar{i}}$, there exists $\tilde{x} \in \hat{X}_{\bar{i}}$ such that $\hat{I}_{\Delta x, M}(f)(x)=f(\tilde{x})$. We then use the Lipschitz property of $f$ and (16) to get the result.

When no truncation is achieved, we have a rate of convergence in $O\left(\Delta x^{M+1}\right)$. When the truncation is achieved, for example $\hat{I}_{\Delta x, M}(f)(x)=\bar{f}_{\bar{i}}$, then

$$
I_{\Delta x, 1} \leq \hat{I}_{\Delta x, M}(f)(x) \leq I_{\Delta x, M}(f)(x),
$$

where $I_{\Delta x, 1}$ correspond to the linear interpolator and then

$$
\left|\hat{I}_{\Delta x, M}(f)(x)-f(x)\right| \leq \max \left(\left|I_{\Delta x, M}(f)(x)-f(x)\right|,\left|I_{\Delta x, 1}(f)(x)-f(x)\right|\right),
$$

so the rate of convergence remains at least equal to 2 .

The third point is easily check by noticing that $I_{\Delta x, M}$ is a Lagrange interpolator so linear, that $I_{\Delta x, M}(m)=m$ and that the truncation operator $\operatorname{tr}$ satisfies $\operatorname{tr}(f+m)=\operatorname{tr}(f)+m$.

Remark 2.3. Some effective interpolation methods such as ENO, WENO can be used for interpolation [18, $27,30]$ while solving Hamilton Jacobi equations but they are not proved convergent. We will show that the previously defined interpolator ensures convergence for Semi Lagrangian schemes and for some Finite Difference schemes but at a rate not better than linear interpolator. The interest of such interpolators will by checked numerically on some examples in the last section. Besides, they are easy to implement independently of the dimension of the problem.

\subsection{A Camilli Falcone style scheme}

The first scheme we study is a modification of the scheme of Camilli and Falcone [11] where the linear interpolator $I_{\Delta x, 1}$ is replaced by a potentially high order interpolator $\hat{I}_{\Delta x, M}$ with $M>1$. We begin by defining the monotone operator $[4,11] \hat{S}$ which is the Lagrangian scheme without interpolation. First for any bounded continuous function $\phi, x, y, z \in \mathbb{R}^{N}$, we set

$$
\begin{gathered}
\hat{S}\left(h, y, t, \phi_{x}\right)=\sup _{\alpha \in \mathcal{A}}\left\{-\frac{1}{h}\left(G\left(h, \alpha, y, \phi_{x}\right)-t\right)+c^{\alpha}(y) t-f^{\alpha}(y)\right\}, \\
G\left(h, \alpha, y, \phi_{x}\right)=\frac{1-h c^{\alpha}(y)}{2 P} \sum_{i=1}^{P}\left(\phi\left(x+h b^{\alpha}(y)+\sqrt{h P} \sigma_{i}^{\alpha}(y)\right)+\phi\left(x+h b^{\alpha}(y)-\sqrt{h P} \sigma_{i}^{\alpha}(y)\right)\right),
\end{gathered}
$$

where $\sigma_{i}^{\alpha}$ is the i-th column of $\sigma$.

The semi discretized scheme $S$ is defined as follows for $y$ a quadrature point.

$$
S\left(h, y, t,[\phi]_{x}\right)=\hat{S}\left(h, y, t,\left(\hat{I}_{\Delta x, M} \phi\right)_{x}\right) .
$$

So the discretized problem leads to find $U$ function on $X_{\Delta x, M}$ such that

$$
\left|\hat{S}\left(h, y, U(y),\left(\hat{I}_{\Delta x, M} U\right)_{y}\right)\right| \leq \epsilon(h, \Delta x), \text { for } y \in X_{\Delta x, M} .
$$

We first recall some results on the solution associated to the semi discretized scheme that can be found in $[4,11]$

Proposition 2.4. Assume that (A1), (A2) hold. Then there exists a unique bounded function $v_{h}$ uniformly in $C^{0,1}\left(\mathbb{R}^{N}\right)$ satisfying

$$
\hat{S}\left(h, x, v_{h}(x),\left(v_{h}\right)_{x}\right)=0 \quad \text { for } x \in \mathbb{R}^{N} .
$$


We next prove that the scheme $S$ satisfies the first assumptions of the article :

Proposition 2.5. Assume (A1) hold and that $\Delta x=h^{q}$. Then the scheme (18) satisfies assumptions (S1), (S2), (S3) with $k_{2}=\min (2 q-1,1), k_{4}=1, p=q-1$.

Proof. First assumption (S2) follows easily from (A1). (S3) follows easily using lemma 2.2: for $v$ regular the consistency error is bounded by

$$
\epsilon(h, \Delta x)=C\left(h\left|\mathcal{D}^{4} v\right|_{0}+h\left|\mathcal{D}^{2} v\right|_{0}+\frac{\Delta x^{2}}{h}\left|\mathcal{D}^{2} v\right|_{0}\right) .
$$

Using (A1), and lemma 2.2, for $m>0$ :

$$
G\left(h, \alpha, y,\left(\hat{I}_{\Delta x, M}(\phi+m)\right)_{x}\right)=G\left(h, \alpha, y,\left(\hat{I}_{\Delta x, M}(\phi)\right)_{x}\right)+\left(1-h c^{\alpha}(x)\right) m,
$$

So

$$
S\left(h, y, t+m,\left[I_{\Delta x, M}(\phi+m)\right]_{x}\right) \geq S\left(h, y, t,\left[I_{\Delta x, M}(\phi)\right]_{x}\right)+2 \lambda m,
$$

and property (8) is checked.

Suppose $v \in C_{b}\left(\mathbb{R}^{N}\right), w \in C^{0,1}\left(\mathbb{R}^{N}\right), v \geq w$. If $x \in \hat{X}_{\bar{i}}$ is such that $\hat{I}_{\Delta x, M}(v)(x) \leq \hat{I}_{\Delta x, M}(w)(x)$ let's introduce $v\left(x_{\bar{i}, \tilde{l}}\right)=\min _{\tilde{j}} v\left(x_{\bar{i}, \tilde{j}}\right)$. It satisfies $v\left(x_{\bar{i}, \tilde{l}}\right) \leq \hat{I}_{\Delta x, M}(v)(x)$ so using lemma 2.2

$$
\begin{array}{r}
\hat{I}_{\Delta x, M}(v)(x)-\hat{I}_{\Delta x, M}(w)(x) \geq v\left(x_{\bar{i}, \tilde{l}}\right)-\hat{I}_{\Delta x, M}(w)(x), \\
\quad \geq v\left(x_{\bar{i}, \tilde{l}}\right)-w\left(x_{\bar{i}, \tilde{l}}\right)-|w|_{1} \Delta x \sqrt{N}, \geq-|w|_{1} \Delta x \sqrt{N} .
\end{array}
$$

So for all $x \in \mathbb{R}^{N}$

$$
\hat{I}_{\Delta x, M}(v)(x) \geq \hat{I}_{\Delta x, M}(w)(x)-|w|_{1} \Delta x \sqrt{N}
$$

and

$$
G\left(h, \alpha, y,\left(\hat{I}_{\Delta x, M}(v)\right)_{x}\right) \geq G\left(h, \alpha, y,\left(\hat{I}_{\Delta x, M}(w)\right)_{x}\right)-\left(1+h\left|c^{\alpha}(y)\right|_{0}\right)|w|_{1} \Delta x \sqrt{N}
$$

So

$$
S\left(h, y, t,\left[\hat{I}_{\Delta x, M}(v)\right]_{x}\right) \leq S\left(h, y, t,\left[\hat{I}_{\Delta x, M}(w)\right]_{x}\right)+\left(1+h\left|c^{\alpha}(y)\right|_{0}\right)|w|_{1} \frac{\sqrt{N} \Delta x}{h}
$$

Similarly if $v \in C^{0,1}\left(\mathbb{R}^{N}\right), w \in C_{b}\left(\mathbb{R}^{N}\right), v \geq w$, noting $w\left(x_{\bar{i}, \tilde{l}}\right)=\max _{\bar{j}} v\left(x_{\bar{i}, \tilde{j}}\right)$, and using lemma 2.2

$$
\begin{array}{r}
\hat{I}_{\Delta x, M}(v)(x)-\hat{I}_{\Delta x, M}(w)(x) \geq \hat{I}_{\Delta x, M}(v)(x)-w\left(x_{\bar{i}, \tilde{l}}\right), \\
\geq v\left(x_{\bar{i}, \tilde{l}}\right)-w\left(x_{\bar{i}, \tilde{l}}\right)-|v|_{1} \Delta x \sqrt{N} \geq-|v|_{1} \Delta x \sqrt{N}
\end{array}
$$

so

$$
\hat{I}_{\Delta x, M}(v)(x) \geq \hat{I}_{\Delta x, M}(w)(x)-|v|_{1} \Delta x \sqrt{N}
$$

and

$$
S\left(h, y, t,\left[\hat{I}_{\Delta x, M}(v)\right]_{x}\right) \leq S\left(h, y, t,\left[\hat{I}_{\Delta x, M}(w)\right]_{x}\right)+\left(1+h\left|c^{\alpha}(y)\right|_{0}\right)|v|_{1} \frac{\sqrt{N} \Delta x}{h}
$$

so that the (6) and (7) properties are checked. 
We need to prove that we can construct an approximate solution of the discretized problem. We introduce the operator $T$ defined for $U$ a function on $X_{\Delta x, M}$ :

$$
\left(T_{h, \Delta x} U\right)(x)=\inf _{\alpha \in \mathcal{A}}\left\{\left(1-h c^{\alpha}(x)\right)\left(\Pi_{\Delta x, h}(U)\right)(x)+h f^{\alpha}(x)\right\} \text { for } x \in X_{\Delta x, M},
$$

where the operator $\Pi_{\Delta x, h}$ is

$$
\left(\Pi_{\Delta x, h} U\right)(x)=\frac{1}{2 P} \sum_{i=1}^{2 P}\left(\left(\hat{I}_{\Delta x, M} U\right)\left(x+h b^{\alpha}(x)+\sqrt{P h} \sigma_{i}^{\alpha}(x)\right)+\left(\hat{I}_{\Delta x, M} U\right)\left(x+h b^{\alpha}(x)-\sqrt{P h} \sigma_{i}^{\alpha}(x)\right)\right) .
$$

We then recursively define $T_{h, \Delta x}^{s}$ for $s \in \mathbb{N}$ and $s \geq 2$ by

$$
\left(T_{h, \Delta x}^{s} U\right)(x)=\left(T_{h, \Delta x}\left(T_{h, \Delta x}^{s-1} U\right)\right)(x) .
$$

Proposition 2.6. Assume (A1), (A2) hold. Suppose that $\Delta x=h^{q}$ with $q>2$. There exists $s \in \mathbb{N}$ depending on $h$ and $C$ independent of $h$ such that $u_{h}=\hat{I}_{\Delta x, M}\left(T_{h, \Delta x}^{s} 0\right)$ is an $\epsilon\left(C h^{q-2}\right)$ solution of scheme $S$.

Proof. Note $v_{h}$ the unique solution given of scheme (19).

For $x \in X_{\Delta x, M}, U$ a function on $X_{\Delta x, M}$, using $|\inf A-\inf B| \leq \sup |A-B|$

$$
\begin{aligned}
\left|\left(T_{h, \Delta x} U\right)(x)-v_{h}(x)\right| \leq & (1-\lambda h) \sum_{i=1}^{2 P}\left(\sup _{\alpha \in \mathcal{A}} \mid\left(\hat{I}_{\Delta x, M} U\right)\left(x+h b^{\alpha}(x)+\sqrt{h P} \sigma_{i}^{\alpha}(x)\right)-\right. \\
& v_{h}\left(x+h b^{\alpha}(x)+\sqrt{h P} \sigma_{i}^{\alpha}(x)\right) \mid \\
& \left.+\left|\left(\hat{I}_{\Delta x, M} U\right)\left(x+h b^{\alpha}(x)-\sqrt{h P} \sigma_{i}^{\alpha}(x)\right)-v_{h}\left(x+h b^{\alpha}(x)+\sqrt{h P} \sigma_{i}^{\alpha}(x)\right)\right|\right),
\end{aligned}
$$

then notice that for $x \in \hat{X}_{\bar{i}}$

$$
\begin{aligned}
\left|\hat{I}_{\Delta x, M}(U)(x)-v_{h}(x)\right| & =\left|\sum_{\tilde{j}} w_{\bar{i}, \tilde{j}}(U)(x)\left(U\left(x_{\bar{i}, \tilde{j}}\right)-v_{h}(x)\right)\right|, \\
& \leq \sum_{j, l} w_{\bar{i}, \tilde{j}}(U)(x)\left|U\left(x_{\bar{i}, \tilde{j}}\right)-v_{h}(x)\right|, \\
& \leq \sup _{\tilde{j}}\left|U\left(x_{\bar{i}, \tilde{j}}\right)-v_{h}(x)\right|, \\
& \leq \sup _{\tilde{j}}\left|U\left(x_{\bar{i}, \tilde{j}}\right)-v_{h}\left(x_{\bar{i}, \tilde{j}}\right)\right|+\Delta x \sqrt{N}\left|v_{h}\right|_{1}, \\
& \leq\left|U-v_{h}\right|_{0}+C \Delta x,
\end{aligned}
$$

where we have use the uniform boundedness of $v_{h}$ in $C^{0,1}\left(\mathbb{R}^{N}\right)$. Gathering (24) and (25) we get

$$
\left|\left(T_{h, \Delta x} U\right)(x)-v_{h}(x)\right| \leq(1-\lambda h)\left(\left|U-v_{h}\right|_{0}+C \Delta x\right) .
$$

Iterating we find that

$$
\left|\left(T_{h, \Delta x}^{k} 0\right)(x)-v_{h}(x)\right| \leq(1-\lambda h)^{k}\left|v_{h}\right|_{0}+C \frac{\Delta x}{h} .
$$

Taking $k=\min \left(i \in \mathbb{N}\right.$ such that $\left.i \geq \frac{(q-1) \log (h)}{\log (1-\lambda h)}\right)$, using the fact that $\Delta x=h^{q}$, we get that

$$
\left|\left(T_{h, \Delta x}^{k} 0\right)(x)-v_{h}(x)\right| \leq C h^{q-1} .
$$


Let's prove that $u_{h}=\hat{I}_{\Delta x, N}\left(T_{h, \Delta x}^{k} 0\right)$ is an $\epsilon\left(h^{q-2}\right)$ solution of the scheme $S$.

As in (25)

$$
\begin{aligned}
\left|\hat{I}_{\Delta x, M}\left(T_{h, \Delta x}^{k} 0\right)(x)-v_{h}(x)\right| & \leq\left|T_{h, \Delta x}^{k} 0-v_{h}\right|_{0}+C \Delta x \\
& \leq C h^{q-1}
\end{aligned}
$$

where we have used (27), so

$$
\left|u_{h}(x)-v_{h}(x)\right| \leq C h^{q-1} .
$$

Then using (A1), the fact that $u_{h}=\hat{I}_{h, \Delta x} u_{h}$, and (28)

$$
\begin{aligned}
\left|S\left(h, x, u_{h}(x),\left[u_{h}\right]_{x}\right)\right| & =\left|\hat{S}\left(h, x, u_{h}(x),\left(u_{h}\right)_{x}\right)-\hat{S}\left(h, x, v_{h}(x),\left(v_{h}\right)_{x}\right)\right|, \\
& \leq \sup _{\alpha \in \mathcal{A}}\left|\frac{1}{h}\left[\left(G\left(h, \alpha, x,\left(u_{h}\right)_{x}\right)-u_{h}(x)\right)-\left(G\left(h, \alpha, x,\left(v_{h}\right)_{x}\right)-v_{h}(x)\right)\right]\right|, \\
& \leq h^{q-2} .
\end{aligned}
$$

Proposition 2.7. Assume (A1), (A2), (A3) hold. Suppose $u_{h}$ has being constructed as in proposition 2.6, the previous developed framework gives us that we can find $q$ such that

$$
\left|u-u_{h}\right|<C h^{\frac{1}{10}}
$$

Proposition 2.8. Assume (A1), (A2) hold. Taking $q \geq \frac{5}{4}, u_{h}$ being constructed as in proposition 2.6, we have

$$
\left|u-u_{h}\right|<C h^{\frac{1}{4}}
$$

Proof. Because we have a bound on $\left|v_{h}-u\right|_{0}$ in $O\left(h^{\frac{1}{4}}\right)$ (see [4]), a direct use of (28) shows

$$
\begin{aligned}
\left|u-u_{h}\right| & \leq\left|u-v_{h}\right|_{0}+\left|v_{h}-u_{h}\right|_{0}, \\
& \leq c\left(h^{\frac{1}{4}}+h^{q-1}\right)
\end{aligned}
$$

giving the result.

Remark 2.9. Propositions 2.7 and 2.8 are generalization of the results of theorem 5.1 and 6.1 in [13] in the almost monotone case.

Remark 2.10. The bound given by the framework is not tight because we introduced a switching system in our approach and not in the latter proposition.

\subsection{Finite Difference scheme}

In this part we suppose that $a^{\alpha}$ is independent of $x$. The matrix $a^{\alpha}$ can be written $P^{\alpha} D^{\alpha}\left(P^{\alpha}\right)^{t}$ where $P^{\alpha}$ is a unitary matrix with $\mathrm{i}$-th columns $\xi_{i}^{\alpha}$ and $D^{\alpha}$ is a diagonal $D_{i, j}^{\alpha}=\delta_{i, j} d_{i}^{\alpha} \geq 0$. We note $\left(b^{\alpha}\right)^{+}(x)$ the vector such that $\left(b^{\alpha}\right)_{i}^{+}(x)=\max \left(0, b^{\alpha_{i}}(x)\right)$ and $\left(b^{\alpha}\right)_{i}^{-}(x)=\max \left(0,-b^{\alpha_{i}}(x)\right)$. The operator $\mathcal{L}^{\alpha}$ can be discretized for 
a regular function $u$ using two parameters $h$ and $\hat{h}$ by

$$
\begin{aligned}
\mathcal{L}^{\alpha}\left(x, u, \mathcal{D} u, \mathcal{D}^{2} u\right) \simeq & \sum_{i=1}^{N} \frac{d_{i}^{\alpha}}{2} \frac{2 u(x)-u\left(x-h \xi_{i}^{\alpha}\right)-u\left(x+h \xi_{i}^{\alpha}\right)}{h^{2}} \\
& -\sum_{i=1}^{N}\left(b^{\alpha}\right)_{i}^{+}(x) \frac{u\left(x+\hat{h} e_{i}\right)-u(x)}{\hat{h}}+\sum_{i=1}^{N}\left(b^{\alpha}\right)_{i}^{-}(x) \frac{u(x)-u\left(x-\hat{h} e_{i}\right)}{\hat{h}} \\
& +c^{\alpha}(x) u(x)-f(x),
\end{aligned}
$$

where $\left(e_{i}\right)_{i=1, N}$ is the canonical basis in $\mathbb{R}^{N}$. We suppose that the equation has been normalized such that

$$
\sup _{\alpha} \sum_{i} d_{i}^{\alpha}+\left|b_{i}^{\alpha}(x)\right| \leq 1
$$

This is always possible because of (A1) and noting that $\sum_{i} d_{i}^{\alpha}=\operatorname{tr}\left[a^{\alpha}\right]$.

For $z \in \mathcal{Q}^{\alpha}=\left\{0,\left\{\xi_{i}^{\alpha}\right\}_{i=1, N},\left\{e_{i}\right\}_{i=1, N}\right\}$ we define the transition probability (see [21]) for $\frac{h^{2}}{h} \leq 1$

$$
\begin{aligned}
& p^{\alpha}(x, x)=1-\sum_{i}\left\{d_{i}^{\alpha}+\left|b_{i}^{\alpha}(x)\right| \frac{h^{2}}{\hat{h}}\right\} \\
& p^{\alpha}\left(x, x \pm \hat{h} e_{i}\right)=\left(b^{\alpha}\right)_{i}^{ \pm}(x) \frac{h^{2}}{\hat{h}}, \\
& p^{\alpha}\left(x, x \pm h \xi_{i}^{\alpha}\right)=\frac{d_{i}^{\alpha}}{2} .
\end{aligned}
$$

For any bounded continuous function $\phi, x, y, z \in \mathbb{R}^{N}$, we define the operator $\hat{S}$

$$
\hat{S}\left(h, y, t, \phi_{x}\right)=\sup _{\alpha \in \mathcal{A}}\left\{-\frac{1}{h^{2}}\left[\sum_{z \in \mathcal{Q}^{\alpha}} p^{\alpha}(y, y+z) \phi(x+z)-t\right]+c^{\alpha}(y) t-f^{\alpha}(y)\right\} .
$$

First we are interested in getting a solution $v_{h}$ of

$$
\hat{S}\left(h, x, v_{h}(x),\left(v_{h}\right)_{x}\right)=0, x \in \mathbb{R}^{N},
$$

in $C^{0,1}\left(\mathbb{R}^{N}\right)$ and get a convergence bound of $\left|u-v_{h}\right|$.

We introduce another assumption stronger than assumption (A2):

Assumption (A4). The constant $\lambda$ in (A1) satisfies $\lambda>\max \left(\sup _{\alpha} \frac{1}{2}\left[\sigma^{\alpha}\right]_{1}^{2}+\left[b^{\alpha}\right]_{1}, 2 \sqrt{N} \sup _{\alpha}\left[b^{\alpha}\right]_{1}\right)$.

By using [4] we get the following proposition

Proposition 2.11. Under assumptions (A1), (A4) the operator $\hat{S}$ is monotone, consistent, and there exists a unique solution $v_{h}$ bounded uniformly in $C^{0,1}\left(\mathbb{R}^{N}\right)$ of equation (30) and u solution of (1) satisfies

$$
\left|u-v_{h}\right| \leq C h^{\frac{1}{2}}
$$

Remark 2.12. We imposed that the $a^{\alpha}$ is independent of $x$ to get a solution $v_{h}$ in $C^{0,1}$ which is necessary for us in order to satisfy (S1). Without this assumption we can't prove that $v_{h}$ is in $C^{0,1}$ : only $L_{\infty}$ bounds on the error are available (see [3]). As an alternative it would have been possible to use Bonnans and al. [10] or Krylov [19] discretization with Krylov [19] convergence results with bounds for $v_{h}$ in $C^{0,1}\left(\mathbb{R}^{N}\right)$. 
We introduce the operator

$$
S\left(h, y, t,[\phi]_{x}\right)=\hat{S}\left(h, y, t,\left(\hat{I}_{\Delta x, M} \phi\right)_{x}\right) .
$$

We give the convergence results obtained with the Finite Difference scheme :

Proposition 2.13. Assume (A1) hold and that $\Delta x=h^{q}, \hat{h}=h$. Then the scheme (32) satisfies assumptions (S1), (S2), (S3) with $k_{2}=\min (1,2 q-2), k_{4}=2, p=q-2$

Proof. First assumption (S2) follows easily from (A1). (S3) follows easily using lemma 2.2: for $v$ regular the consistency error is bounded by

$$
\epsilon(h, \Delta x)=C\left(h^{2}\left|\mathcal{D}^{4} v\right|_{0}+\hat{h}\left|\mathcal{D}^{2} v\right|_{0}+\Delta x^{2}\left(\frac{1}{h^{2}}+\frac{1}{\hat{h}}\right)\left|\mathcal{D}^{2} v\right|_{0}\right)
$$

Using the fact that $\sum_{z \in \mathcal{Q}^{\alpha}} p^{\alpha}(y, y+z)=1$ with positive weights,

$$
S\left(h, y, t+m,\left[I_{\Delta x, M}(\phi+m)\right]_{x}\right)=S\left(h, y, t,\left[I_{\Delta x, M}(\phi)\right]_{x}\right),
$$

and property (8) is checked.

Suppose $v \in C_{b}\left(\mathbb{R}^{N}\right), w \in C^{0,1}\left(\mathbb{R}^{N}\right), v \geq w$, using estimate $(21)$, the fact that $\sum_{z \in \mathcal{Q}^{\alpha}} p^{\alpha}(y, y+z)=1$ with positive weights,

$$
S\left(h, y, t,\left[\hat{I}_{\Delta x, M}(v)\right]_{x}\right) \leq S\left(h, y, t,\left[\hat{I}_{\Delta x, M}(w)\right]_{x}\right)+|w|_{1} \frac{\sqrt{N} \Delta x}{h^{2}}
$$

Similarly if $v \in C^{0,1}\left(\mathbb{R}^{N}\right), w \in C_{b}\left(\mathbb{R}^{N}\right), v \geq w$ using estimate (22),

$$
S\left(h, y, t,\left[\hat{I}_{\Delta x, M}(v)\right]_{x}\right) \leq S\left(h, y, t,\left[\hat{I}_{\Delta x, M}(w)\right]_{x}\right)+|v|_{1} \frac{\sqrt{N} \Delta x}{h^{2}},
$$

so that the (6) and (7) properties are checked.

We then have to check that we can find an $\epsilon$ solution $u_{h}$ satisfying (4). We introduce the operator defined for $U$ a function on $X_{\Delta x, M}$ : for $x \in X_{\Delta x, M}$

$$
\left(T_{h, \Delta x} U\right)(x)=\inf _{\alpha \in \mathcal{A}}\left\{\frac{1}{1+h^{2} c^{\alpha}(x)} \sum_{z \in \mathcal{Q}} p^{\alpha}(x, x+z) \hat{I}_{\Delta x, M}(U)(x+z)+h^{2} f^{\alpha}(x)\right\},
$$

and $T_{h, \Delta x}^{s}$ operator is still defined by equation (23).

Proposition 2.14. Assume (A1), (A4) hold. Suppose that $\Delta x=h^{q}$ with $q>2, \hat{h}=h$. There exists $s \in \mathbb{N}$ depending on $h$ and $C$ independent of $h$ such that $u_{h}=\hat{I}_{h, \Delta x}\left(T_{h, \Delta x}^{s} 0\right)$ is an $\epsilon\left(C h^{q-4}\right)$ solution of scheme $S$.

Proof. The proof is similar to the one of proposition 2.6. We first prove that

$$
\left|\left(T_{h, \Delta x}^{k} 0\right)(x)-v_{h}(x)\right| \leq \frac{1}{\left(1+\lambda h^{2}\right)^{k}}\left|v_{h}\right|_{0}+C \frac{\Delta x}{h^{2}} .
$$

Taking $k=\min \left(i \in \mathbb{N}\right.$ such that $\left.i \geq-\frac{(q-2) \log (h)}{\log \left(1+\lambda h^{2}\right)}\right)$, using the fact that $\Delta x=h^{q}$, we get that

$$
\left|\left(T_{h, \Delta x}^{k} 0\right)(x)-v_{h}(x)\right| \leq C h^{q-2} .
$$


Let's prove that $u_{h}=\hat{I}_{\Delta x, N}\left(T_{h, \Delta x}^{k} 0\right)$ is an $\epsilon\left(h^{q-4}\right)$ solution of the scheme $S$.

As in (25),

$$
\begin{aligned}
\left|\hat{I}_{\Delta x, M}\left(T_{h, \Delta x}^{k} 0\right)(x)-v_{h}(x)\right| & \leq\left|T_{h, \Delta x}^{k} 0-v_{h}\right|_{0}+C \Delta x \\
& \leq C h^{q-2}
\end{aligned}
$$

where we have used (35), so

$$
\left|u_{h}(x)-v_{h}(x)\right| \leq C h^{q-2} .
$$

Then using (A1), (36), the relation $|\sup A-\sup B| \leq \sup |A-B|$, the fact that the probabilities are between 0 and 1 with sum equal to 1 and the fact that $u_{h}=\hat{\hat{I}}_{h, \Delta x} u_{h}$ :

$$
\begin{aligned}
\left|S\left(h, x, u_{h}(x),\left[u_{h}\right]_{x}\right)\right| & =\left|\hat{S}\left(h, x, u_{h}(x),\left(u_{h}\right)_{x}\right)-\hat{S}\left(h, x, v_{h}(x),\left(v_{h}\right)_{x}\right)\right| \\
& \leq \frac{1}{h^{2}}\left|u_{h}-v_{h}\right|_{0} \\
& \leq c^{q-4} .
\end{aligned}
$$

Proposition 2.15. Assume (A1), (A4), (A3) hold. Constructing an $\epsilon$ solution of (4) with the $u_{h}$ given by proposition 2.14, we get

$$
\left|u-u_{h}\right|_{0} \leq h^{\frac{1}{5}}
$$

with $q$ above $21 / 5$.

Proof. The rate of convergence and the value $q=\frac{21}{5}$ is due to a direct use of theorem 1.10.

Proposition 2.16. Assume (A1), (A4) hold. Using $u_{h}$ given by proposition 2.14, we get

$$
\left|u-u_{h}\right|_{0} \leq h^{\frac{1}{2}}
$$

Proof. This is a direct use of (36) with the rate of convergence $\left|u-v_{h}\right|_{0} \leq h^{\frac{1}{2}}$ of proposition 2.11 taking $q=\frac{5}{2}$.

\section{Numerical tests}

The theoretical bounds obtained in the previous section are not better than the ones obtained with linear interpolation. The interest of this approximation relies on the fact that where the solution is smooth we expect that the solution won't be truncated and that the consistency error will be far better than the theoretical one. All case treated are two dimensional cases. For the first three examples, we only give results for the Semi-Lagrangian scheme because the Finite Difference scheme developed coincides with the classical Finite Difference. The domain linked to the resolution of equation (1) will be noted $Q, \mathbb{1}$ is the diagonal unitary matrix and 1 is the vector with 1 components. For all Semi-Lagrangian Schemes we choose $h=0.0002$. We discretized the one dimensional space of controls $\mathcal{A}$ with 2000 controls. The software is parallelized with 48 cores as explained in [32]. The interpolation used are either linear (2 points per mesh in each direction, monotone scheme), or quadratic (3 points per mesh in each direction), or Cubic (4 points per mesh in each direction). On all the cases and all the tests the fixed point iteration is converging but it can be very slow especially for Finite 
Differences. The maximum number of iterations is taken equal to 100000 and no acceleration was used. The stopping criterion between iteration $i$ and $i+1$ was

$$
\left|u^{i+1}-u^{i}\right| \leq 10^{-7}
$$

The different schemes are stable and numerically convergent. The numerical order of convergence does not show a regular behavior so it has not been reported. In the table Err is for the $\mathcal{L}^{\infty}$ norm for a given discretization given by a number of mesh ( the same in each direction), while ItN gives the number of fixed point iteration used and the computational time is given in seconds.

\subsection{A first regular test case}

The solution of this test case is given by

$$
u(x, y)=\sin (\pi x) \sin (\pi y)
$$

The coefficients are given by

$$
c_{a}(t, x)=C, \quad \sigma_{a}(t, x)=\sigma a \mathbb{1}, \quad b^{\alpha}=b 1,
$$

and the function $f^{\alpha}$ is given by

$$
f^{\alpha}(x, y)=\left(C+\pi^{2} \sigma^{2} 1_{u(x, y)>0}\right) u(x, u)-b \pi(\cos (\pi x) \sin (\pi y)+\sin (\pi x) \cos (\pi y)) .
$$

The numerical coefficients are

$$
Q=[0,2]^{2}, \quad b=0.3, \quad c=0.55, \quad \sigma=1, \quad A=[0,1]
$$

and we use a zero Dirichlet boundary condition. Results are given in table 1 and this first regular case clearly indicates that the quadratic approximation is by far the most efficient interpolation : even on this regular case the use of a cubic interpolator doesn't decrease the error with a CPU time multiplied at least three-fold. In fact it is rapidly converging to the $h$ discretized operator so that the interpolation error becomes negligible for a number of mesh equal to 80 .

TABle 1 . Test case 1

\begin{tabular}{|c|c|c|c|c|c|c|c|c|c|}
\hline \# mesh & \multicolumn{3}{|c|}{ LINEAR } & \multicolumn{3}{c|}{ QUADRATIC } & \multicolumn{3}{c|}{ CUBIC } \\
\hline & Err & ItN & Time & Err & ItN & Time & Err & ItN & Time \\
10 & 1.169 & 25900 & 138 & 0.051 & 29483 & 919 & 0.183 & 45108 & 5182 \\
20 & 1.028 & 26555 & 468 & 0.0065 & 29398 & 3619 & 0.0083 & 29486 & 10770 \\
40 & 0.758 & 27211 & 1879 & 0.0012 & 29485 & 12633 & 0.0011 & 29706 & 43371 \\
80 & 0.243 & 28076 & 6923 & 0.0003 & 29621 & 50988 & 0.0003 & 29788 & 175163 \\
160 & 0.103 & 28620 & 27762 & 0.0003 & 29748 & 19517 & & & \\
320 & 0.018 & 29282 & 108000 & & & & & & \\
\hline
\end{tabular}

\subsection{A second regular problem}

The solution is here again

$$
u(x, y)=\sin (\pi x) \sin (\pi y)
$$




$$
c_{a}(t, x)=C, \quad \sigma_{a}(t, x)=\sigma a \mathbb{1}, \quad b^{\alpha}=b\left(a, \sqrt{1-a^{2}}\right), a \in[\underline{a}, \bar{a}] .
$$

Noting

$$
\begin{gathered}
\tilde{a}=\frac{\sin (\pi y) \cos (\pi x)}{\sqrt{\sin (\pi x)^{2} \cos (\pi y)^{2}+\cos (\pi x)^{2} \sin (\pi y)^{2}}}, \\
\phi(a)=a \sin (\pi y) \cos (\pi x)+\sqrt{1-a^{2}} \sin (\pi x) \cos (\pi y),
\end{gathered}
$$

for $b \leq 0$, the function $f^{\alpha}$ is here given by

$$
f^{\alpha}(x, y)=\left(C+\pi^{2} \sigma^{2}\right) u(x, u)-b \pi K,
$$

where $K$ is the maximum of $\phi(\underline{\mathrm{a}}), \phi(\bar{a})$ and $\phi(\tilde{a})$ conditionally to $\underline{\mathrm{a}} \leq \tilde{a} \leq \bar{a}$. We take $\underline{\mathrm{a}}=-1, \bar{a}=1, \sigma=1$, $C=0.6, b=-1, Q=\left[0, \frac{1}{2}\right]$. As boundary condition we take the Dirichlet value given by $u$.

Results obtained in table 2 still show the superiority of the quadratic interpolation for this regular problem with exactly the same conclusions.

TABLE 2. Test case 2

\begin{tabular}{|c|c|c|c|c|c|c|c|c|c|}
\hline \# mesh & \multicolumn{3}{|c|}{ LINEAR } & \multicolumn{3}{c|}{ QUADRATIC } & \multicolumn{3}{c|}{ CUBIC } \\
\hline & Err & ItN & Time & Err & ItN & Time & Err & ItN & Time \\
8 & 0.1195 & 493 & 2 & 0.0115 & 1304 & 33 & 0.012 & 1571 & 99 \\
16 & 0.0632 & 903 & 13 & 0.0022 & 1318 & 100 & 0.0022 & 1589 & 390 \\
32 & 0.0191 & 1197 & 50 & 0.0003 & 1319 & 405 & 0.0003 & 1590 & 1574 \\
64 & 0.0062 & 1278 & 207 & & & & & & \\
\hline
\end{tabular}

\subsection{A non regular problem}

We keep the same notations as in the subsection 3.2 . Introducing

$$
\begin{gathered}
\tilde{a}=\frac{\frac{1}{2} \sin (\pi y) \cos \left(\frac{1}{2} \pi x\right)}{\sqrt{\sin (0.5 \pi x)^{2} \cos (\pi y)^{2}+\frac{1}{4} \cos (0.5 \pi x)^{2} \sin (\pi y)^{2}}}, \\
\hat{a}=-\tilde{a}, \\
\hat{\phi}(a)=\frac{1}{2} a \sin (\pi y) \cos \left(\frac{1}{2} \pi x\right)+\sqrt{1-a^{2}} \sin \left(\frac{1}{2} \pi x\right) \cos (\pi y),
\end{gathered}
$$

for $b \leq 0$, the function $f$ is then

$$
f^{\alpha}= \begin{cases}\left(C+\pi^{2} \sigma^{2}\right) \sin (\pi y) \sin (\pi x)-b \pi K & \text { for }-1 \leq x \leq 0 \\ \left(C+\pi^{2} \sigma^{2} \frac{5}{8}\right) \sin \left(\frac{1}{2} \pi x\right) \cos (\pi x)-b \pi \hat{K} & \text { for } 0 \leq x \leq 1\end{cases}
$$

where $\hat{K}$ is the maximum between $\hat{\phi}(\underline{\mathrm{a}}), \hat{\phi}(\bar{a})$ and $\hat{\phi}(\tilde{a})$ conditionally to $\underline{\mathrm{a}} \leq \tilde{a} \leq \bar{a}, \hat{\phi}(\hat{a})$ conditionally to $\underline{\mathrm{a}} \leq \hat{a} \leq \bar{a}$. We take the values $\underline{\mathrm{a}}=-1, \bar{a}=1, \sigma=1, C=0.6, b=-1, Q=[-1,1]^{2}$.

The boundary conditions are given by the values of the following function $\psi$ :

$$
\psi(x, y)=\sin (\pi y) \begin{cases}\sin (\pi x) & \text { pour }-1 \leq x \leq 0 \\ \sin \left(\frac{1}{2} \pi x\right) & \text { pour } 1 \geq x \geq 0\end{cases}
$$

This test case is interesting because the continuous function $\psi$ is a regular solution for $x<0$ and for $x>0$ but it turns out that it is not the viscosity solution $u$ of the problem. The reference solution (an estimation of $u$ ) is numerically calculated with a quadratic interpolation with 128 meshes per directions. All the methods seems to converge towards the same solution but here again the superiority of the quadratic interpolation is obvious. 
TABLE 3. Test case 3

\begin{tabular}{|c|c|c|c|c|c|c|c|c|c|}
\hline \# mesh & \multicolumn{3}{|c|}{ LINEAR } & \multicolumn{3}{c|}{ QUADRATIC } & \multicolumn{3}{c|}{ CUBIC } \\
\hline & Err & ItN & Time & Err & ItN & Time & Err & ItN & Time \\
8 & 0.970 & 1810 & 6 & 0.10 & 9406 & 239 & 0.097 & 8877 & 536 \\
16 & 0.909 & 3256 & 44 & 0.0141 & 10546 & 777 & 0.0132 & 10677 & 2553 \\
32 & 0.767 & 5551 & 214 & 0.00363 & 10942 & 3277 & 0.0030 & 10916 & 10469 \\
64 & 0.436 & 8259 & 1313 & 0.00187 & 10984 & 12107 & 0.0004 & 10980 & 41952 \\
128 & 0.140 & 8854 & 5348 & & & & & & \\
256 & 0.0478 & 10386 & 26056 & & & & & & \\
\hline
\end{tabular}

\subsection{A last problem for degeneracy of the diffusion operator}

The last test case will allow us to test the Finite Difference method with a diffusion operator which is degenerated : The solution is here again

$$
u(x, y)=\sin (\pi x) \sin (\pi y)
$$

The coefficients are given by

$$
c_{a}(t, x)=C, \quad \sigma_{a}(t, x)=\sigma\left(\begin{array}{l}
1 \\
a
\end{array}\right), \quad b^{\alpha}=b\left(\begin{array}{l}
1 \\
a
\end{array}\right),
$$

and the function $f^{\alpha}$ is given by

$$
\begin{aligned}
f^{\alpha}(x, y)= & \sup _{\hat{a} \in[\underline{a}, \bar{a}]}\left(\left(C+\pi^{2} \sigma^{2}\left(1+\hat{a}^{2}\right)\right) u(x, u)\right. \\
& \left.-\left(\sigma^{2} \pi^{2}(\cos (\pi x) \cos (\pi y)+\pi b \sin (\pi x) \cos (\pi y))\right) \hat{a}\right) \\
& -b \pi \cos (\pi x) \sin (\pi y) .
\end{aligned}
$$

The values taken are $\underline{\mathrm{a}}=-1, \bar{a}=1, \sigma=1, C=0.7, b=0.5, Q=[-1,1]^{2}$. The results clearly indicate that the Finite Difference method proposed is not competitive with the Semi Lagrangian scheme. The convergence of the fixed point iteration for a step $h=0.01$ is not achieved with Finite Difference with 100000 iterations (the error given between the last two iterations is around $2.10^{-7}$ ): there is certainly no exact solution to the scheme. Once again, the Semi Lagrangian scheme with quadratic interpolation is the most effective method.

Special thank to anonymous referees for their corrections and suggestions.

\section{REFERENCES}

[1] Remi Abgrall. Construction of simple, stable, and convergent high order schemes for steady first order Hamilton-Jacobi equations. SIAM Journal on Scientific Computing, 31(4):2419-2446, 2009.

[2] Steeve Augoula and Rémi Abgrall. High order numerical discretization for Hamilton-Jacobi equations on triangular meshes. Journal of Scientific Computing, 15(2):197-229, 2000.

[3] Guy Barles and Espen R Jakobsen. Error bounds for monotone approximation schemes for Hamilton-Jacobi-Bellman equations. SIAM journal on numerical analysis, 43(2):540-558, 2005.

[4] Guy Barles and Espen Robstad Jakobsen. On the convergence rate of approximation schemes for Hamilton-Jacobi-Bellman equations. ESAIM: Mathematical Modelling and Numerical Analysis, 36(1):33-54, 2002.

[5] Guy Barles and Panagiotis E Souganidis. Convergence of approximation schemes for fully nonlinear second order equations. Asymptotic analysis, 4(3):271-283, 1991.

[6] Olivier Bokanowski, Maurizio Falcone, Roberto Ferretti, Lars Grüne, Dante Kalise, and Hasnaa Zidani. Value iteration convergence of $\epsilon$-monotone schemes for stationary Hamilton-Jacobi equations. Discrete and Continuous Dynamical Systems-Series A, 35(9):4041-4070, 2015. 
TABLE 4. Test case 4

\begin{tabular}{|c|c|c|c|c|c|c|c|c|c|}
\hline \multicolumn{10}{|c|}{ Semi Lagrangian } \\
\hline \# mesh & \multicolumn{3}{|c|}{ LINEAR } & \multicolumn{2}{c|}{ QUADRATIC } & \multicolumn{3}{c|}{ CUBIC } \\
\hline & Err & ItN & Time & Err & ItN & Time & Err & ItN & Time \\
8 & 0.958 & 1298 & 2 & 0.126 & 12879 & 176 & 0.117 & 17817 & 564 \\
16 & 0.838 & 3448 & 20 & 0.0227 & 14066 & 576 & 0.0147 & 18854 & 2347 \\
32 & 0.639 & 6841 & 653 & 0.0024 & 14326 & 2314 & 0.0015 & 19088 & 9430 \\
64 & 0.154 & 13004 & 4825 & 0.0018 & 14429 & 8499 & & & \\
\hline \hline \multicolumn{10}{|c|}{ Finite Difference } \\
\hline \multicolumn{10}{|c|}{$h=0.01$} \\
\hline mesh & \multicolumn{10}{|c|}{ LINEAR } & \multicolumn{10}{c|}{ QUADRATIC } & \multicolumn{3}{|c|}{ CUBIC } \\
\hline 8 & Err & ItN & Time & Err & ItN & Time & Err & ItN & Time \\
16 & 0.9598 & 8258 & 38 & 0.200 & $10^{5}$ & 1649 & 0.139 & $10^{5}$ & 7168 \\
32 & 0.9276 & 15240 & 257 & 0.064 & $10^{5}$ & 4986 & 0.0345 & $10^{5}$ & 28590 \\
64 & 0.8517 & 26815 & 1307 & 0.023 & $10^{5}$ & 19450 & 0.0129 & $10^{5}$ & 113792 \\
128 & 0.69840 & 46830 & 8787 & 0.008 & $10^{5}$ & 145509 & & & \\
256 & 0.4599 & 79658 & 49851 & & & & & & \\
\hline
\end{tabular}

[7] Olivier Bokanowski, Maurizio Falcone, and Smita Sahu. An efficient filtered scheme for some first order time-dependent Hamilton-Jacobi equations. SIAM Journal on Scientific Computing, 38(1):A171-A195, 2016.

[8] Olivier Bokanowski, Nadia Megdich, and Hasnaa Zidani. Convergence of a non-monotone scheme for Hamilton-Jacobi-Bellman equations with discontinous initial data. Numerische Mathematik, 115(1):1-44, 2010.

[9] Olivier Bokanowski, Athena Picarelli, and Christoph Reisinger. High-order filtered schemes for time-dependent second order HJB equations. ESAIM: Mathematical Modelling and Numerical Analysis, 52(1):69-97, 2018.

[10] J Frédéric Bonnans, Élisabeth Ottenwaelter, and Housnaa Zidani. A fast algorithm for the two dimensional HJB equation of stochastic control. ESAIM: Mathematical Modelling and Numerical Analysis, 38(4):723-735, 2004.

[11] Fabio Camilli and Maurizio Falcone. An approximation scheme for the optimal control of diffusion processes. ESAIM: Mathematical Modelling and Numerical Analysis, 29(1):97-122, 1995.

[12] Kristian Debrabant and Espen Jakobsen. Semi-lagrangian schemes for linear and fully non-linear diffusion equations. Mathematics of Computation, 82(283):1433-1462, 2013.

[13] Kristian Debrabant and Espen R Jakobsen. Semi-lagrangian schemes for linear and fully non-linear Hamilton-Jacobi-Bellman equations. arXiv preprint arXiv:1403.1217, 2014.

[14] Lawrence C Evans and Avner Friedman. Optimal stochastic switching and the Dirichlet problem for the Bellman equation. Transactions of the American Mathematical Society, 253:365-389, 1979.

[15] Maurizio Falcone and Roberto Ferretti. Semi-Lagrangian approximation schemes for linear and Hamilton-Jacobi equations, volume 133. SIAM, 2013.

[16] Brittany D Froese and Adam M Oberman. Convergent filtered schemes for the Monge-Ampere partial differential equation. SIAM Journal on Numerical Analysis, 51(1):423-444, 2013.

[17] Max Jensen and Iain Smears. On the convergence of finite element methods for Hamilton-Jacobi-Bellman equations. SIAM Journal on Numerical Analysis, 51(1):137-162, 2013.

[18] Guang-Shan Jiang and Danping Peng. Weighted eno schemes for Hamilton-Jacobi equations. SIAM Journal on Scientific computing, 21(6):2126-2143, 2000.

[19] Nicolai V Krylov. The rate of convergence of finite-difference approximations for Bellman equations with Lipschitz coefficients. Applied Mathematics \&3 Optimization, 52(3):365-399, 2005.

[20] NV Krylov. On the rate of convergence of finite-difference approximations for Bellman equations with variable coefficients. Probability theory and related fields, 117(1):1-16, 2000.

[21] Harold Kushner and Paul G Dupuis. Numerical methods for stochastic control problems in continuous time, volume 24. Springer Science \& Business Media, 2013.

[22] Olga Lepsky. Spectral viscosity approximations to Hamilton-Jacobi solutions. SIA M journal on numerical analysis, 38(5):14391453,2000

[23] P-L Lions and PE Souganidis. Convergence of muscl and filtered schemes for scalar conservation laws and Hamilton-Jacobi equations. Numerische Mathematik, 69(4):441-470, 1995. 
[24] José-Luis Menaldi. Some estimates for finite difference approximations. SIAM journal on control and optimization, 27(3):579$607,1989$.

[25] Adam M Oberman. Convergent difference schemes for degenerate elliptic and parabolic equations: Hamilton-Jacobi equations and free boundary problems. SIAM Journal on Numerical Analysis, 44(2):879-895, 2006.

[26] Adam M Oberman and Tiago Salvador. Filtered schemes for Hamilton-Jacobi equations: A simple construction of convergent accurate difference schemes. Journal of Computational Physics, 284:367-388, 2015.

[27] Stanley Osher and Chi-Wang Shu. High-order essentially nonoscillatory schemes for Hamilton-Jacobi equations. SIAM Journal on numerical analysis, 28(4):907-922, 1991.

[28] David M Pooley, Peter A Forsyth, and Ken R Vetzal. Numerical convergence properties of option pricing PDEs with uncertain volatility. IMA Journal of Numerical Analysis, 23(2):241-267, 2003.

[29] Alfio Quarteroni, Riccardo Sacco, and Fausto Saleri. Numerical mathematics, volume 37. Springer Science \& Business Media, 2010.

[30] Chi-Wang Shu. High order numerical methods for time dependent Hamilton-Jacobi equations. In Mathematics and computation in imaging science and information processing, pages 47-91. World Scientific, 2007.

[31] Iain Smears and Endre Süli. Discontinuous galerkin finite element methods for time-dependent Hamilton-jacobi-Bellman equations with cordes coefficients. Numerische Mathematik, 133(1):141-176, 2016

[32] Xavier Warin. Some non-monotone schemes for time dependent Hamilton-Jacobi-Bellman equations in stochastic control. Journal of Scientific Computing, 66(3):1122-1147, 2016. 